\title{
Design for a high resolution electron energy loss microscope
}

\author{
Marian Mankos, Khashayar Shadman \\ Electron Optica Inc., 1000 Elwell Court Ste.110, Palo Alto, California 94303, USA
}

Raphaël Hahn, Yan J. Picard and Daniel Comparat

Laboratoire Aimé Cotton, CNRS, Univ. Paris-Sud, ENS Paris Saclay,

Université Paris-Saclay, Bât. 505, 91405 Orsay, France

Olena Fedchenko, Gerd Schönhense

Johannes Gutenberg Universität Mainz, Inst. für Physik, 55128 Mainz

Lionel Amiaud, Anne Lafosse

Institut des Sciences Moléculaires d'Orsay (ISMO), CNRS,

Univ Paris-Sud, Université Paris-Saclay, F-91405 Orsay, France

Nick Barrett

SPEC, CEA, CNRS, Université Paris-Saclay, CEA Saclay, 91191 Gif-sur-Yvette Cedex, France

(Dated: September 14, 2019)

\begin{abstract}
An electron optical column has been designed for High Resolution Electron Energy Loss Microscopy (HREELM). The column is composed of electron lenses and a beam separator that are placed between an electron source based on a laser excited cesium atom beam and a time-of-flight (ToF) spectrometer or a hemispherical analyzer (HSA). The instrument will be able to perform full field low energy electron imaging of surfaces with sub-micron spatial resolution and meV energy resolution necessary for the analysis of local vibrational spectra. Thus, non-contact, real space mapping of microscopic variations in vibrational levels will be made possible. A second imaging mode will allow for the mapping of the phonon dispersion relations from microscopic regions defined by an appropriate field aperture.
\end{abstract}

PACS numbers:

\section{INTRODUCTION}

Vibrational levels carry the signature of local chemistry and they are responsible for many functional properties. In this context, mapping the vibrational and electronic spectra with high spatial resolution (tens of nanometers) on a surface would represent a real breakthrough in surface science and in characterization techniques.

There is, at present, no non-scanning technical solution for imaging electron energy losses with low energy electrons and hence mapping local, microscopic phonon variations with both high spatial and spectroscopic resolution. By including a low bandwidth (meV) electron source based on laser excitation of an atomic beam, a high resolution detector, appropriate electron optics and stable power supplies, an energy loss microscope has been designed that is capable of mapping the vibrational losses with $5 \mathrm{meV}$ energy resolution and $15-20 \mathrm{~nm}$ spatial resolution by probing the sample with electrons with $0-20$ $\mathrm{eV}$ incident energy.

The challenge of this design is to combine monochromatic, low energy electrons with very high spectroscopic, spatial and momentum resolution. Methods exist for the different aspects of this challenge taken individually, but none offer the global solution that this invention will allow. Here the different state of the art techniques are briefly reviewed.
Phonon dispersion can be measured by neutron scattering and, in certain conditions, by X-rays [1], however, neither are particularly surface sensitive. High Resolution Electron Energy Loss Spectroscopy (HREELS) is the only method capable of mapping the phonon dispersion at the surface, however, it has no microscopic spatial resolution. The classic HREELS set-up requires a monochromator for the source and for the detection, each with a $127^{\circ}$ deflection geometry [2]. The best reported resolution with such set-up is $1.3 \mathrm{meV}$ [3]. A typical example is the phonon dispersion of $\mathrm{SrTiO}_{3}$ using $6 \mathrm{eV}$ electrons [4] and an energy resolution of $60 \mathrm{meV}$. Tipenhanced Raman spectroscopy (TERS) with scanning near-field microscopy can perform Raman imaging at the nanoscale. Maps are typically several hundred nm wide and the best reported spatial resolution is now below $30 \mathrm{~nm}$ [5]. However, there are three distinct drawbacks. First, the measurement is sequential and is achieved by scanning, rather than parallel, full-field acquisition. Navigation on an unknown sample surface must therefore be done by another method or the position to be analysed must be precisely known beforehand, which is usually not the case. Second, tip-surface interactions can influence the measurement. Finally, TERS is a real-space imaging mode without angular sensitivity, as a result it cannot measure the dispersion relations.

The use of electron optics allows for the measurement 
of the electron wave vector. Low energy electron microscopy (LEEM) [6, 7] was developed in the late 1950s and early 1960 s thanks to the use of stainless steel vacuum chambers that provided a ultra-high vacuum environment. A variety of electron sources have been used, notably $\mathrm{LaB}_{6}$ cathodes, field emission guns (FEG), or even single atom sources. However, the energy spread is no better than $0.25 \mathrm{eV}$, which excludes resolution of vibrational structure. The best reported spatial resolution in standard LEEM with $\mathrm{LaB}_{6}$ electron source is about $15 \mathrm{~nm}$. The resolution improves to below $10 \mathrm{~nm}$ with a FEG and to $2 \mathrm{~nm}$ with aberration correction [8]. LEEM is sensitive to potential variations, surface charge and chemistry.

An all-magnetic monochromator/spectrometer system can allow sub-5 meV energy-resolution electron energyloss spectroscopy in the scanning transmission electron microscope as demonstrated recently by the Nion company $[9,10]$. The instrument combines a monochromator/spectrometer system at a primary voltage of 100-200 $\mathrm{kV}$. The use of a monochromator, however, significantly increases acquisition time and as the instrument works in transmission, it is only bulk sensitive and requires elaborate sample preparation. In turn, the sample preparation may even modify some of the vibrational states. Radiation induced changes under high energy electron beams can also be an issue in many functional materials [11].

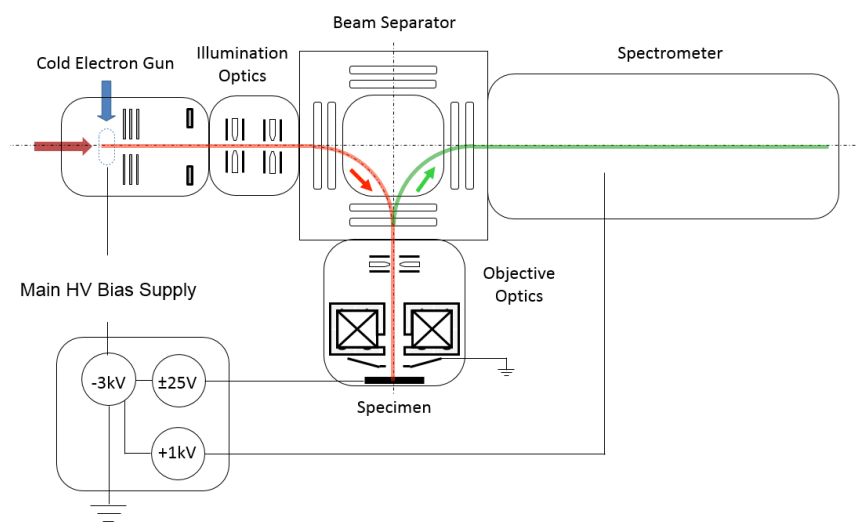

FIG. 1: HREELM column layout, the Cs atoms arriving from the effusion cell are indicated by the red arrow, the ionizing laser beam by the vertical blue arrow. The solid green line indicates the path of the electrons from the source through the illumination optics and beam separator (small red arrow) to the sample and the backscattered electron path (small green arrow) to the spectrometer. The microscope floats at $3 \mathrm{kV}$, the start volatge can be adjusted over $25 \mathrm{~V}$.

The aim of the HREELM project is to develop a spectroscopic low energy electron microscope with energy resolution in the few meV range. The instrument will allow high resolution electron energy loss surface spectroscopy with $\sim 20 \mathrm{~nm}$ spatial resolution. In addition, it will allow for the mapping of the phonon dispersion from sub micron regions.

The LEEM column incorporates a cold electron source based on laser ionization of suitable Rydberg states of Cs atoms emitted by an effusion cell. Figure 1 shows the layout of the instrument. Cold electrons are accelerated in the electron gun and collimated by the illumination optics before transfer into the beam separator. Relatively low voltage $(3 \mathrm{kV})$ power supplies will be used with $10^{-6}$ ripple in order not to compromise the required meV resolution. The beam separator, a magnetic prism array, is an energy-dispersive element that deflects the incoming electrons by $90^{\circ}$ into the objective optics. The objective optics transports the electron beam toward the specimen, located in an immersion objective lens. The immersion lens decelerates the electron beam and illuminates the sample with a low energy electron beam. Electrons scattered by the specimen are reaccelerated in the opposite direction, and transported towards the beam separator. The beam separator deflects the incoming electron again by $90^{\circ}$, this time into a HSA or ToF analyzer, as shown in Fig. 1, with meV energy resolution for detection. The electrons are slowed down in the spectrometer to increase the dispersion. Its voltage is floating on the common high-voltage (HV) supply to reduce electrical ripples and thus to improve the spectroscopic resolution.

In a conventional LEEM, the real image of the specimen is transferred into the achromatic plane of the dispersive beam separator, while the diffraction pattern is transferred into its slit plane, which has an energy dispersion of typically a few $\mu \mathrm{m} / \mathrm{eV}$. This (albeit relatively small) energy dispersion in the diffraction pattern would be detrimental to the performance of the energy analyzer. The key point of the present design is that the LEEM optics must be capable of switching between two modes, the (conventional LEEM) image mode, where the real image of the specimen is placed in the achromatic plane of the beam separator, and the diffraction mode, where the diffraction pattern of the specimen is placed in the achromatic plane of the beam separator. This switching requirement is not present in a conventional LEEM, and drives the additional complexity of the illumination and objective optics. In a conventional LEEM, typically two lenses are sufficient for the illumination optics, and one lens is needed in the objective optics. In the design presented here, the illumination optics requires two more field lenses, and the objective optics requires three more lenses to maintain the flexibility of the illumination optics. Here and throughout the article the term "diffraction image" is synonymous with "momentum image" i.e. the momentum distribution of the scattered electrons. The positions of the real and diffraction images in the key planes of the beam separator are discussed in detail in section IV and illustrated in figs. 7 and 8. In addition, this swap must be carried out without changing the objective lens, to maintain stable sample imaging conditions and diffraction patterns. This requirement couples the objective optics to the illumination optics, thereby complicating the design.

The paper is organized as follows. Section II presents the methodology and analysis tools employed. Section 
III describes possible cold electron sources and energy analyzers. Section IV details the illumination, prism, and objective optics, as well as the time of flight detector. Finally, section $\mathrm{V}$ presents the overall performances, which include the combined impact of the optical aberrations with the electron-electron, Coulomb interactions.

\section{METHODOLOGY}

The HREELM electron-optical column was designed in three phases: electron-optical concept, preliminary and detailed component design. In the concept phase, the overall column layout and the individual components were defined. During the preliminary component design phase, the first order optics of the column was examined by using a thin lens model. In the detailed component design phase, cross-sectional drawings of the components suitable for prototype design were produced. Here, the primary optics and the third order aberrations of the various components were computed. In the analysis, both electric and magnetic lenses were considered as potential candidates. Electrostatic lenses, however, were found to produce excessive aberrations, precluding a compact design for the overall column. Therefore, magnetic lenses were incorporated throughout. In the final step, the optical properties of all the components were combined along with the impact of the Coulomb interactions to assess the optical performance of the instrument.

For the simulations, the electron-optical software packages developed by MEBS, Ltd. were utilized. In particular, the SOFEM program, a second order finite element method field solver, was used to compute the fields; the PRISM2 program was used to compute the optical properties and aberrations of the magnetic prism; and the IMAGE program was used to perform Monte Carlo simulations of the electron beam to naturally combine the impact of Coulomb interactions with the lens aberrations.

\section{ELECTRON SOURCE AND ENERGY-DISPERSING ANALYSER}

In this section, the technical solutions envisaged for an electron beam compatible with the aforementioned requirements and the consequences in terms of instrument design, especially for the energy-dispersing element at the end of the electron-optical system, are presented. As mentioned, other high performance instruments rely heavily on the use of monochromators for the production of monoenergetic electron beams [12-16]. To avoid high voltages, low-current and stability issues the instrument was designed without a monochromator, relying instead on an intrinsically narrow bandwidth electron source such as the one pioneered by Gallagher and York in 1974 [17] using ionization of an atomic beam emitted from an effusive oven. Even at sub-meV kinetic energies, electron velocities are around $15 \mathrm{~km} / \mathrm{s}$ which far exceeds atomic velocities. Thus the atomic temperature (around $400 \mathrm{~K}$ ) does not increase the energy dispersion of the electrons.

With the appropriate ionizing laser, the source can be operated in continuous or in pulsed mode. The analyzer element at the end of the electron-optical system should, of course, be compatible with the meV energy width provided by the source. It can either be an imaging ToF spectrometer or a conventional energy-dispersing element, e.g. a magnetic prism or a HSA. All have been employed in momentum-imaging instruments [18-21].

In this study, two different detection schemes have been investigated. The energy loss spectra can be recorded using a ToF spectrometer or by a HSA. Both the HSA and the TOF can be floated at the microscope potential and therefore integrated into the system without major technical problems. For both types of analyzers, the resolution-sensitive potentials of the dispersing element (for the HSA: Herzog potential, outer sphere, and inner sphere; for the ToF: potential of drift tube) have to float on approximately $3 \mathrm{kV}$. Thus, to achieve meV resolution the voltage modules must have a precision of $10^{-6}$, accessible given the ripple / noise and drift values for ultra-high precision modules proposed by various manufacturers. The choice of the analyzer impacts the design of the electron source and vice-versa. Owing to its capability to record a full energy spectrum in parallel, the ToF method has a higher detection efficiency than window-type spectrometers. However, it requires a pulsed source and is typically limited to low flux of $10 \mathrm{pA}$ whereas the HSA solution can be used with high current (in the $\mathrm{nA}$ range) and continuous sources.

The energy resolution of the HSA of radius $R$ is given by $\Delta E=w \mathrm{E}_{\text {pass }} / 2 R$, where $\mathrm{E}_{\text {pass }}$ is the pass energy and $w$ is the slit width. A $5 \mathrm{meV}$ energy resolution can be obtained with a hemisphere of radius $100 \mathrm{~mm}$, a slit width of $200 \mu \mathrm{m}$, and a pass energy of $5 \mathrm{eV}$. Such a slit size represents a significant challenge for the design of the optical column, but a larger (and so more expensive) HSA can also be used. As only a selected energy slice is recorded at a time, a higher electron flux is required to acquire the $2 \mathrm{D}$ images for all energies in a reasonable time. The HSA thus requires a high-current electron source (in the $\mathrm{nA}$ range) to obtain a full field of view (FoV) image with $5 \mathrm{meV}$ resolution and $\sim 1 \mathrm{eV}$ spectral range.

On the other hand, an imaging ToF spectrometer does not require slits; it records the $2 \mathrm{D}$ position and energies simultaneously thanks to a drift tube and a time-resolving image detector (delay-line detector, DLD) $[21,22]$. The entrance plane is thus an isochrone surface with a zoom lens defining the desired field of view in momentum space. The energy resolution is determined by the drift energy $E_{\mathrm{d}}$ in the ToF section (of length $L$ ) $\mathrm{d} E / \mathrm{d} \tau=-2\left(2 E_{\mathrm{d}}^{3} / \mathrm{m}_{\mathrm{e}}\right)^{1 / 2} / \mathrm{L} ; \mathrm{d} \tau$ being the time resolution $\sim 150 \mathrm{ps}$ of the DLD. Apart from $L$, this equation does not contain any geometry factors. This is different from the situation using a hemispherical spectrometer, where the slit width and the angular spread pose a high challenge for meV resolution and the transfer of a com- 
plete image through the analyzer. The phase-space confinement produced by the entrance slit of a hemispherical analyzer is replaced by the demand for sufficiently precise timing conditions between the source and the detector. For pulsed excitation sources, Liouville's theorem (phase-space conservation) is in favour of imaging ToF instruments, owing to their acceptance of larger phasespace volumes in the 4D parameter landscape of energy and momentum vector of the scattered electrons.

The ToF spectrometer has already been explored and used for a detailed characterization of a cold electron beam from a Cs Magneto-Optical trap (MOT) source demonstrating an energy resolution in the meV range as shown in Fig. 2. The temporal width of $560 \mathrm{ps}$ is not at the limit of the DLD resolution but still allows for ToF imaging with an energy resolution in the meV range [23].

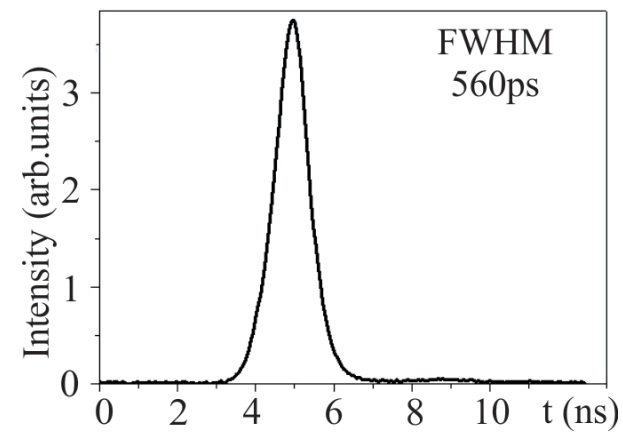

FIG. 2: Time-of-flight spectrum of a pulsed cold electron beam from magneto-optically trapped Cs atoms pumped into the $7 \mathrm{~s}$ state. A Ti-sapphire laser oscillator running at $80 \mathrm{MHz}$ was tuned exactly to the ionization threshold of the cold Cs atoms. This constitutes a pulsed source with $4 \mathrm{meV}$ energy bandwidth.

However, the DLD cannot tolerate multi-hits; therefore $\mathrm{a} \sim 10-100 \mathrm{~ns}$ time separation is required between detected electrons. Thanks to its capability to record a full energy spectrum (typically $1 \mathrm{eV}$ wide) in parallel, the ToF can nevertheless record the image $(\sim 1000 \times 1000$ pixels and $5 \mathrm{meV}$ resolution) in few minutes using a pulsed source of only tens of pA.

In HREELS, about 1 out of 10 of the reflected electrons may lose energy by excitation of vibrating dipole (this is a typical ratio between elastic and inelastic losses). Therefore the acquisition time needed will be an order of magnitude longer, i.e. a few minutes. The typical ratio between incident and backscattered electrons is 10-100 to 1 , therefore an incident current of less than $1 \mathrm{nA}$ should be sufficient, well within the current limits discussed below.

Threshold photo-ionization electron sources have already produced monochromatic beams with energy width below $2 \mathrm{meV}$ [25-27]. To reach this range of energy width requires careful shielding of electric and magnetic fields and control of surface potential drifts. Several limitations on the producible current exists. The main source current limitation comes from the atomic flux and the

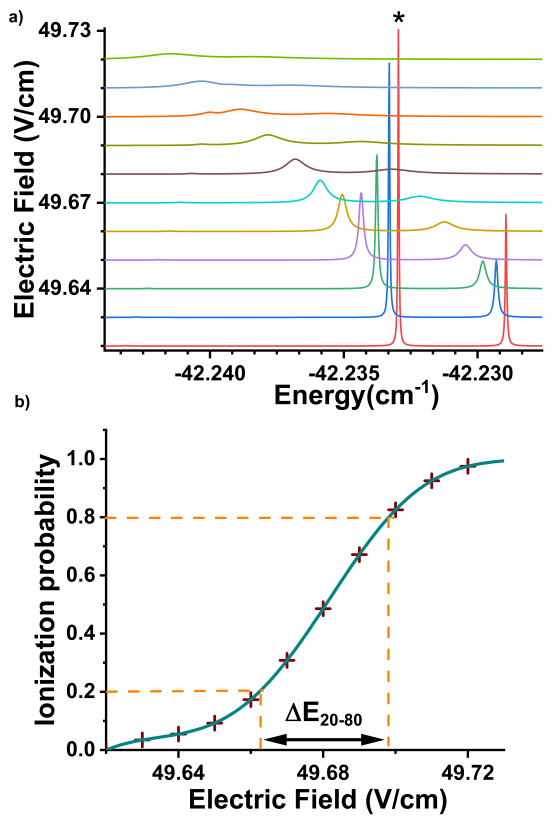

FIG. 3: a) Theoretical calculations of cesium photoabsorption spectra of $\pi$ polarized light from an s state, based on Ref. [24] in presence of an electric field. The horizontal axis is the binding energy. The star indicates the state that is considered in the text and in panel b. b) Ionization probability of the star-marked state as a function of the electric field. Crosses are the state bandwidth extracted from a)

, the line is an interpolation of this data. See text for details of calculations.

ionization efficiency. Indeed, if inside a $2 r \sim 50 \mu \mathrm{m} \mathrm{di-}$ ameter zone, a standard Cs atomic beam produces a flux of $6 \times 10^{12} \mathrm{at} / \mathrm{s}$ [28] with atomic velocity $v \sim 300 \mathrm{~m} / \mathrm{s}$, the small photoionization cross section $\left(\sim 10^{-18} \mathrm{~cm}^{2}\right)$ would require kilowatts-laser power focused on an sub-mm ionization spot for full ionization of the atomic beam. Therefore high currents may be hard to achieve. This can be circumvented by using a laser cavity to locally enhance the laser power, or by increasing the atomic density by slowing the atoms down (a guided 2D-MOT but with flux of $\sim 5 \times 10^{10}$ at/s [29-32] reaches atomic beam velocities of few $\mathrm{m} / \mathrm{s}$ ) or via active compression [33].

An alternative solution is to directly excite an autoionizing Rydberg state with a lifetime in the ns range (such as the one depicted in fig.3) in a well defined electric field. The excitation cross section is higher by orders of magnitude compared to direct photoionization and may allow production of an electron current up to $I \sim 10 \mathrm{nA}[29]$. Spectral resonance conditions in an electric field gradient will select only a specific spatial zone where the state can be excited. This can lead to an ionization zone smaller than the laser spot and can have advantages compared to simple (above threshold) photoionisation. Indeed, in a standard photoionization source, the extraction field used is rather small, about $10-100 \mathrm{mV} / \mathrm{cm}$ [27] in order to reduce the energy width $(\Delta V=E \Delta z)$ to the $\mathrm{meV}$ 
range for a $\Delta z \sim 0.1-1 \mathrm{~mm}$ ionization spot. This low extraction field has the drawback that the ion velocity given by the field is not large enough to extract the ions from the ionization zone, producing a volume charge density $\rho=\frac{I}{v \pi r^{2}}$ that creates an additional $\Delta V \sim \frac{\rho}{\epsilon_{0}} \Delta z$ energy broadening for the electron source. For typical effusive beam velocity, the energy spread versus the current is $\Delta V(\mathrm{meV}) \sim 15 I(\mathrm{nA})[17,26]$. Both direct photoionization and autoionization of Rydberg are potential monochromatic electron sources, the definitive choice requires some more detailed investigation into the currentenergy broadening trade-off and optimization.

For beam currents useful for high resolution analysis with a HSA $(I \sim 1 \mathrm{nA})$ the energy broadening is greater than the required resolution whereas the photoionizing source seems perfectly compatible with a ToF analyzer because it uses pulsed low current sources that can easily attain $<5$ meV energy widths.

For a HSA analyzer, the Rydberg forced field ionization source is envisaged [34]. Indeed, by using Rydberg states that exhibit fast ionization rate changes under an electric field gradient (see fig. 3), it would be possible to have a sub-micron axial ionization zone, which keeps the energy width in the meV range even for quite large extraction fields of $E=10-100 \mathrm{~V} / \mathrm{cm}[35,36]$.

The study of the energy broadening due to the ionic space charge in this case is beyond the scope of this article, but it might be reduced compared to direct laser photoionization, because Rydberg field ionization occurs at a given total (external + space charge) electric field. Therefore the spatial location of the ionization depends also on the ionic space charged effect. Another, possibly detrimental effect comes from the presence of the longitudinal gradient $E^{\prime}$ required to field ionize the moving atoms which implies the existence of a radial field gradient. This creates a potential difference between electrons on the optical axis and at a distance $r$ from the axis [34], given by $\Delta V=\left(3 r^{2} / 8\right) E^{\prime}$. For an ionization radius of $r \sim 100 \mu \mathrm{m}$, a meV-range energy width limits the maximum field gradient to $E^{\prime} \sim 100 \mathrm{~V} / \mathrm{cm} / \mathrm{cm}$.

Our preliminary theoretical studies indicate that an energy width of $\Delta V<10 \mathrm{meV}$ is attainable by placing the atom in a Rydberg state (similar to the one shown in Fig. 3) that quickly ionizes once it enters a sufficiently strong field gradient.

The optimal values of the electric field and of the electric field gradient remain to be determined; however, the design of the electron gun is optimized around the expected values and is sufficiently flexible to ensure only minor revisions during the next steps of the development of the electron source. Indeed, using the present design of the electron gun (see figure 5), the performance of the HREELM column has been simulated with fields as low as $5 \mathrm{~V} / \mathrm{cm}$, which is compatible with the photoionization source, as well as with an extraction field of $50 \mathrm{~V} / \mathrm{cm}$, which is compatible with a Rydberg field ionization source (gradient ranging from $10 \mathrm{~V} / \mathrm{cm} / \mathrm{cm}$ at the centre of the extraction electrodes (antisymmetry plane) to $150 \mathrm{~V} / \mathrm{cm} / \mathrm{cm}$ at $1 \mathrm{~mm}$ axial distance). In the following section, the latter configuration is presented but the results are similar for lower extraction fields. Both configurations are compatible with either the ToF or the HSA analyzer.

\section{ELECTRON OPTICS}

The detailed layout of the prototype HREELM column including the cold electron gun, illumination optics, beam separator prism array, objective optics and ToF spectrometer is shown in Fig. 4. In this section, an overview of the design of each subsystem (electron gun, illumination optics, prism and objective lens) is presented.

\section{A. Electron gun}

Figure 5 shows a schematic of the electron gun, the potential contours, and the axial potential profile for a typical focusing condition. The electron gun comprises five electrodes, all with bore diameters of $1 \mathrm{~mm}$. The first two electrodes form the extractor. For all the sources discussed in the previous section, the electrons are emitted close to the asymmetry plane, located $2 \mathrm{~mm}$ from the extraction electrodes. These electrodes are biased $\pm 10 \mathrm{~V}$ with respect to the beam voltage of $-3 \mathrm{kV}$ in order to apply a weak uniform electric field to the source electrons. The weak field guides the electrons into the column without imparting a large energy spread. The extraction electrodes are followed by three more electrodes that form the gun lens. The focus electrode operates within $1 \mathrm{kV}$ of the beam voltage while the surrounding two electrodes are at ground potential.

Starting at the source location in the mid-plane between the two extractors, i.e. the location of the potential antisymmetry plane, the fundamental rays were computed for the aforementioned gun voltage configuration. The axial ray is shown in Fig. 7. The profile of this ray illustrates that the gun images the source twice, with the second image placed at the center of the first field lens of the illumination optics where the electron source is magnified by a factor of $1.2 \times$. The other fundamental ray, the field ray, which is not shown, determines where the source cross-over is imaged. The location of this plane is highlighted in fig. 7 .

Figure 6 shows the spot intensity distributions in the image and crossover planes formed by the gun lens. These profiles were derived from a model of the electron source, which was guided by the findings of Sec. III. In this model, the electrons have a starting kinetic energy of $1 \mathrm{meV}$ along the positive $\mathrm{z}$ axis and are in a uniform, weak accelerating field so all will be extracted. The emission characteristics of the electron source are characterized by a Gaussian lateral spatial distribution of $100 \mu \mathrm{m}$ FWHM, a Lambertian angular distribution, and a Maxwell-Boltzmann energy distribution with $1 \mathrm{meV}$ 


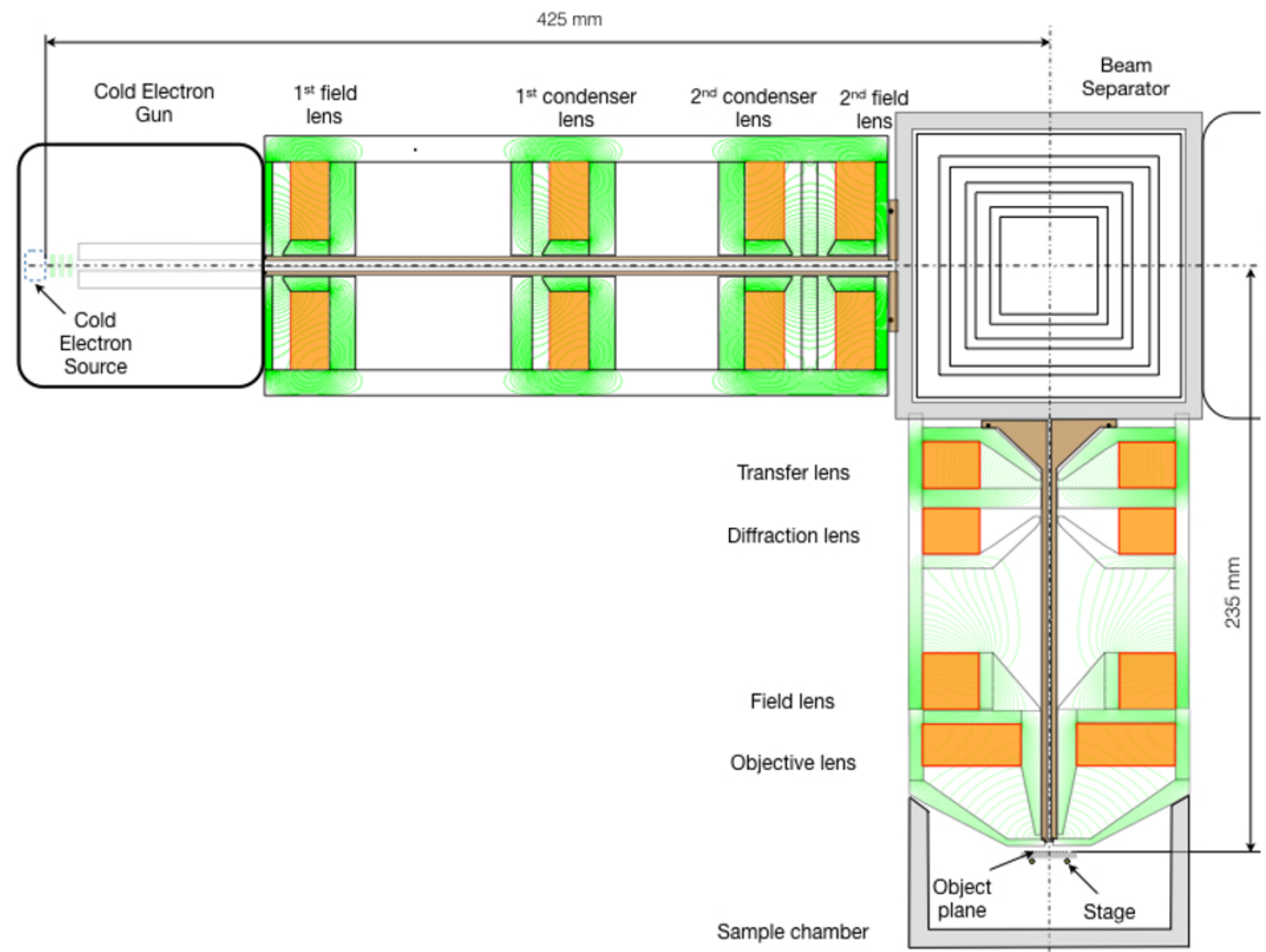

FIG. 4: Proposed detailed prototype layout of the HREELM column showing the different mechanical sub-systems: electron gun; illumination optics; prism and immersion lens.

FWHM. For the simulations, a conservative value of 100 $\mu \mathrm{m}$ has been chosen for the effective lateral source size. This value is comparable to standard tungsten thermal electron emitters, which have been used widely in electron microscopy (TEM and LEEM). The effective lateral source size is determined by the focused spot size of the ionizing laser, and in principle can be reduced into the range of a few tens of $\mu \mathrm{m}$, which would make it comparable to conventional $\mathrm{LaB}_{6}$ electron sources. The axial spread of the source electrons has been neglected since ray-tracing simulations show that the impact of this spread on the optical performance is negligible. The increase in blur due to the partial spatial coherence at high spatial frequencies is not expected to play a role for our resolution target of approximately $20 \mathrm{~nm}$. While the Lambertian distribution is broad and ranges from 0 to 90 degrees, the low energy spread of order $1 \mathrm{meV}$ and the subsequent beam acceleration to $3 \mathrm{keV}$ will collimate the angular distribution by a factor equal to the square root of the ratio of these values, i.e. by more than 3 orders of magnitude into the $10^{-1} \mathrm{mrad}$ range, a value common in TEM.

As seen in Fig. 6, the intensity distribution is approximately Gaussian in the image plane and approximately flat (top-hat like) in the crossover plane. The uniform profiles makes the source crossover the chosen plane to be mapped onto the sample. This mapping is partly accomplished by the first field lens, whose action does not impact the axial ray, allowing for independent control over the placements of the source image and crossover planes.

\section{B. Illumination}

The illumination optics transports the cold electron source to and from the sample via a magnetic prism, which bends the beam by $90^{\circ}$. The illumination col- 


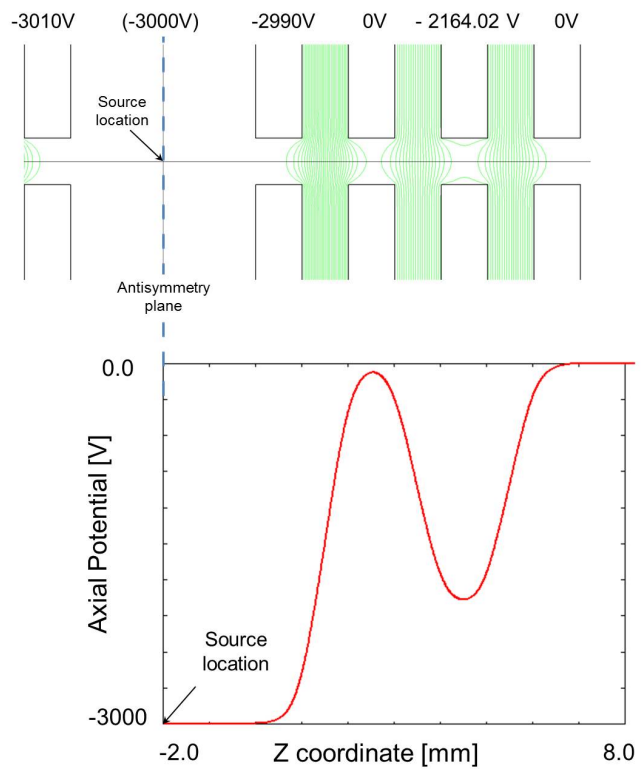

FIG. 5: Overall equipotential distribution of the cold electron gun for typical operating conditions
Image plane

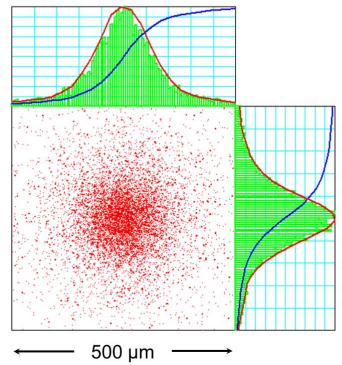

Virtual crossover plane

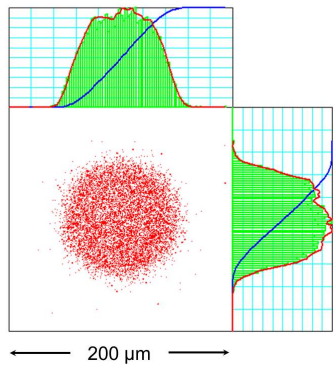

FIG. 6: Final spot shape formed by the cold electron gun in the image (left) and crossover (right) planes

umn has two arms: the source arm, which transports the source electrons to the prism; and the objective arm, which transports the electrons from the prism to the sample and back. The optics are required to illuminate the sample with a uniform, collimated beam and $10 \times$ optical zoom; that is, a FoV at the sample that ranges from 10-100 $\mu \mathrm{m}$. The uniformity is achieved by mapping the uniform source profile at the crossover onto the sample plane. Collimation is achieved by mapping the small Gaussian spot of the source image onto the back-focal (diffraction) plane of the objective lens, which translates to a small divergence angle at the sample. These conditions must be met regardless of the mode of operation, of which there are two: image mode, whereby the sample plane is imaged onto the detector; and diffraction mode, whereby the sample diffraction pattern in the back-focal plane of the objective lens, is imaged onto the detector. Whichever plane is chosen must first be imaged onto the prism achromatic plane, located virtually at the center of the prism. Otherwise, the dispersion imparted to the beam by the prism would introduce an energy dependent shift in the detected image, pushing the beam off-center of the detector.

For ease of analysis and operation, the gun and objective lenses are held fixed throughout; that is, they remain fixed while changing the FoV or when switching the mode. The freedom for optical zoom is derived from a combination of the field and condenser lenses in the source arm.

Figure 7 shows the ray diagram in image mode. Here, the objective arm of the illumination optics maps the sample plane onto the prism achromatic plane. Given that the source crossover must always be mapped onto the sample, the source arm of the illumination optics maps the source crossover onto the achromatic plane. The transfer lens in the objective arm then maps the crossover onto the center of the field lens, which is subsequently mapped onto the sample by the objective lens. Concurrently, the Gaussian image of the source is mapped by the $2^{\text {nd }}$ condenser lens in the source arm onto the prism slit (dispersion) plane, which is conveniently transported with unit magnification onto the slit plane in the objective arm. The field lens in the objective arm then maps the source image onto the objective lens back-focal plane.

The optical zoom from the large to the small FoV is obtained by concurrently exciting the $1^{\text {st }}$ condenser lens and de-exciting the $1^{\text {st }}$ field lens while adjusting the $2^{\text {nd }}$ condenser and field lenses. In the process, the source image is magnified onto the slit plane by $\mathrm{M} \sim 0.06 \times$ and $0.7 \times$ for the large and small FoVs, respectively. The spot in the achromatic plane is de-magnified by $\sim 17 \times$ in the objective arm to produce the correct FoV at the sample plane.

Figure 8 shows the ray diagram in diffraction mode. Here, the objective arm of the illumination optics must map the objective lens back-focal plane onto the prism achromatic plane. Given that the source image must always be mapped onto the objective lens back-focal plane, the source arm of the illumination optics maps, in concert, the source image onto the achromatic plane. Concurrently, the source crossover is mapped by the $1^{\text {st }}$ field lens onto the prism slit plane, which is transported onto the slit plane in the objective arm. Here, the diffraction lens maps the crossover onto the field lens so that, once again, the objective lens maps the crossover onto the sample.

The optical zoom from the large to the small FoV is obtained by concurrently exciting the $1^{\text {st }}$ condenser lens and de-exciting both field lenses while maintaining the $2^{\text {nd }}$ condenser lens de-excited. In the process, the source image is magnified onto the prism achromatic plane by $\mathrm{M} \sim 0.6 \times$ and $2.5 \times$ for the large and small FoVs, respectively. The spot in the slit plane is de-magnified by $\sim 2 \times$ in the objective arm to produce the correct FoV at the sample plane. 


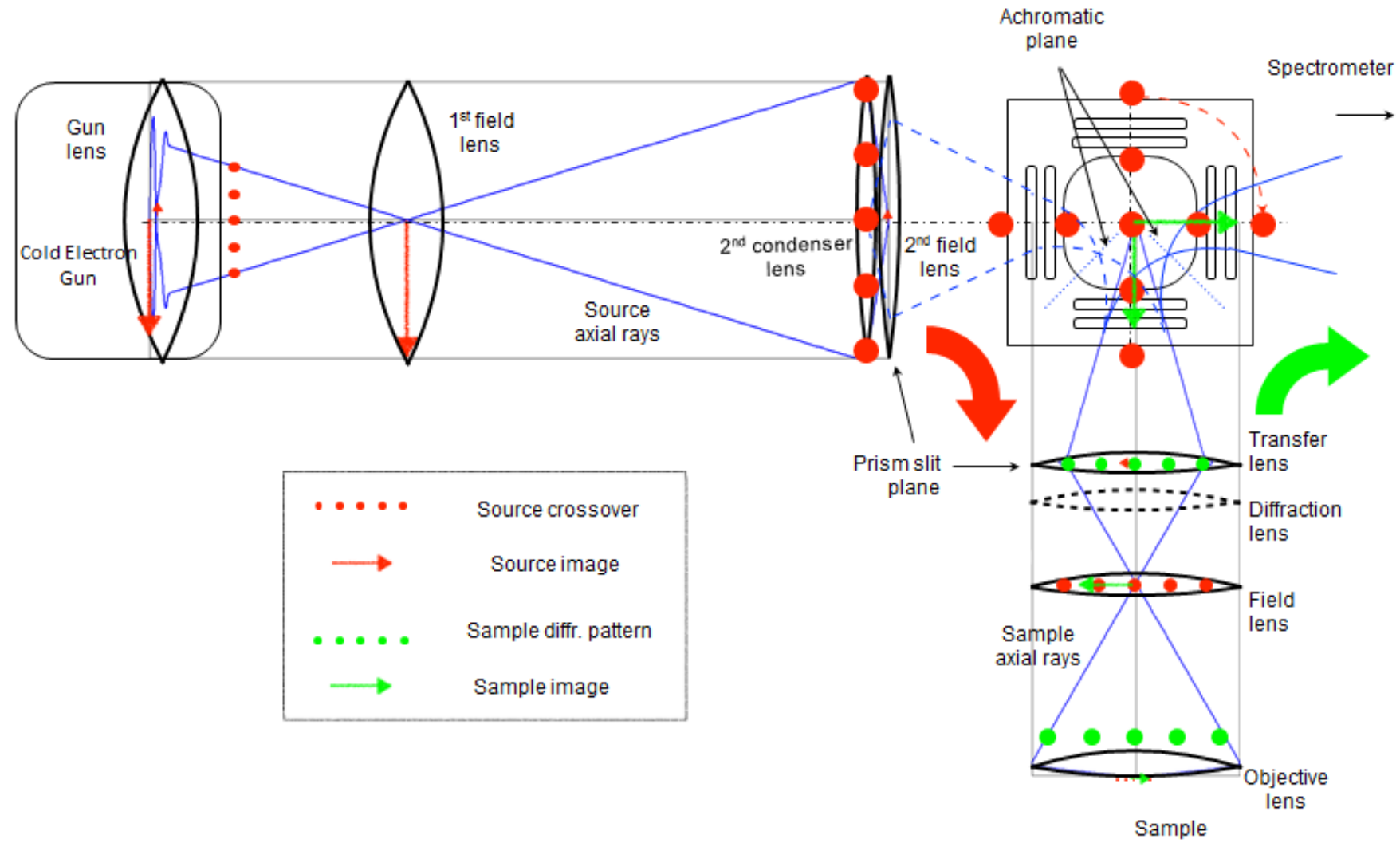

FIG. 7: Ray diagram of the combined illumination and objective optics in image mode. The optical simulation program assumes a straight optical axis so the achromatic plane is virtually at the center of the prism but is physically located at the 45 degree symmetry plane.Straight colorred arrows show image planes, rows of five dots, diffraction planes.

\section{Prism}

The beam separator will be based on a magnetic prism array, a proven and reliable design, which has been developed for low energy electron microscopes to separate the otherwise overlapping illumination and projection optics [37]. The optical properties of magnetic prisms differ considerably in the plane that cuts across the top and bottom magnetic plates (the vertical plane, i.e. perpendicular to the paper) and the plane that separate these two plates (the horizontal or mid-plane, in the plane of the paper in Figs. 7 and 8), giving rise to large astigmatism and distortion [37]. For the LEEM column, the magnetic prism needs to concurrently provide a deflection of $90^{\circ}$ while behaving as a stigmatic round lens in order to preserve the quality of the illuminating beam. This can be achieved in a beam separator that is composed of a close-packed magnetic prism array. The array consists of a large, central square region with a uniform magnetic field between the top and bottom plates, surrounded by several smaller sectors with individual coils to locally modulate and correct the magnetic field. The strengths of these fringe fields are tuned to match the focus in the vertical plane, achieved naturally by the curvature of the path in the mid-plane. The diffraction/slit plane is located outside the prism, while the achromatic plane is located virtually at the center of the prism, and physically at the $45^{\circ}$ quadrant midplane. In this optimized prism array, each quadrant deflects the beam by $90^{\circ}$ and transfers stigmatically the two key planes, the diffraction (slit) and (achromatic) image plane, with unit magnification.

\section{Objective Lens}

The objective lens is an immersion lens with overlapping electrostatic and magnetic fields as shown in Fig. 9. The electron beam, collimated by the illumination optics, is decelerated to the desired landing energy $(0-20 \mathrm{eV})$ at the sample surface where they are scattered and then reaccelerated to the beam transport energy of $3 \mathrm{keV}$. Analogous to the case for the gun lens, the objective lens maps the sample plane onto the center of the field lens to allow for the independent tuning of the diffraction pattern in the objective lens back-focal plane. At the field lens, the image of the sample is magnified by $8.5 \times$. 


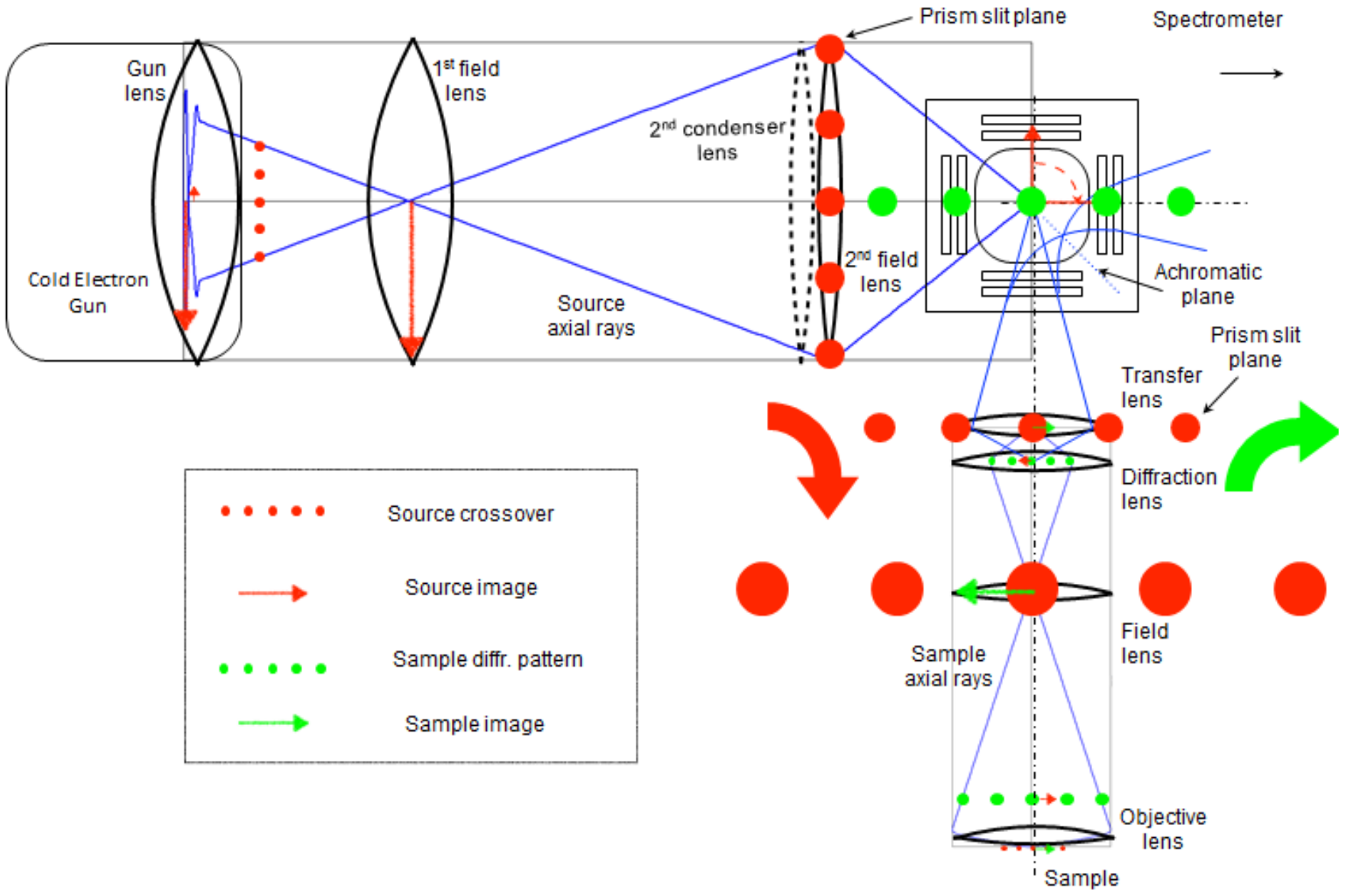

FIG. 8: Ray diagram of combined illumination and objective optics in diffraction mode. The optical simulation program assumes a straight optical axis so the achromatic plane is virtually at the center of the prism but is physically located at the 45 degree symmetry plane.
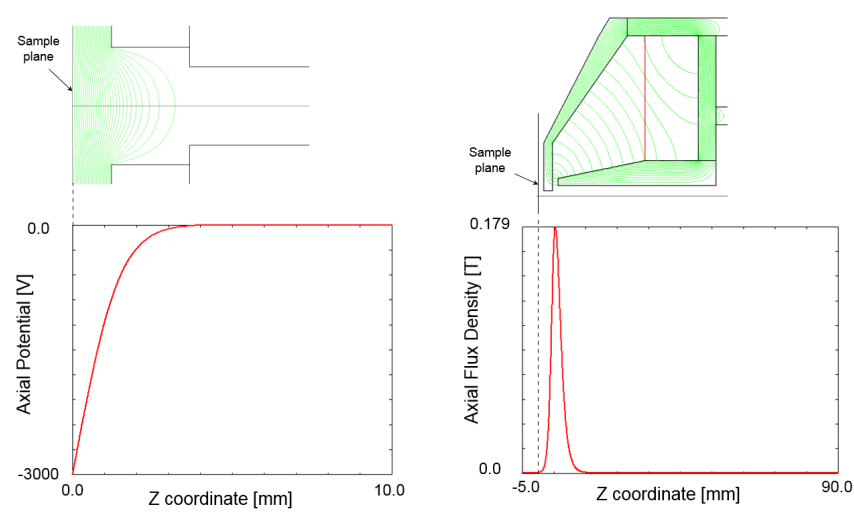

FIG. 9: Prototype layout of the objective optics combining the distribution of the electric potential (left) and the magnetic flux density (right).

\section{OPTICAL PERFORMANCE}

The optical performance of the HRLEEM column was assessed with the IMAGE package from MEBS, Ltd.. The software package computes electron-optical properties by propagating bunches of particles through realistic electromagnetic fields by accurate direct ray-tracing. It captures the combined effects of Coulomb interactions and lens aberrations.

The purpose of the illumination arm, which delivers the beam from the source to the sample, is to produce a spatially uniform spot on the sample over a field of view that is much larger than the typical geometric optical blurs introduced by the aberrations of the lenses involved. Consequently, the aberrations introduced in the source arm are of little consequence. Hence, the analysis is centered on the optical performance of the projection arm, which delivers the beam from the sample to the spectrometer (ToF or HSA) via the objective arm and the prism.

An illustration of the beam spots within the field of 
view of the field lens resulting from the travel of the beam from the sample through the objective lens is shown in Fig. 10. From the contour plots, the geometrical optical blur and distortion in the spot are calculated. These calculations are performed for both modes, image and diffraction, and for the small and large FoVs at the sample (10 and $100 \mu \mathrm{m}$, respectively). The resulting blur and distortion values are then compared to the pixel size. For this purpose, the detector is assumed to have 500 pixels on each side, which translates to a pixel size of 20 and $200 \mathrm{~nm}$ in the sample plane for the small and large FoVs, respectively.
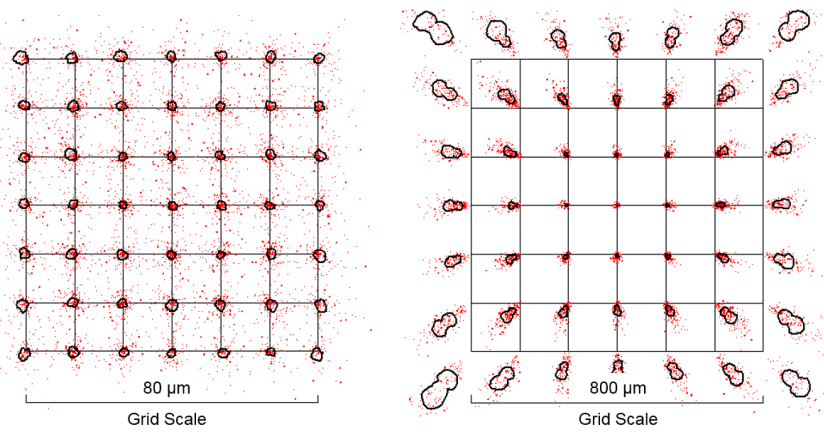

FIG. 10: Spot shapes (20-80 percent rise) for the objective lens only, in the center of the field lens, in image mode for both small and large FoVs.

\section{A. Objective arm}

The simulations in the objective arm start at the sample surface. The electron distribution at the sample is modeled to incorporate the wide angles of the reflected electrons near zero energy. Hence, the electrons are modeled to leave the sample surface with a uniform spatial distribution spanning the appropriate FoV, a Gaussian angular distribution with a FWHM of $500 \mathrm{mrad}$, a start energy of $0.1 \mathrm{eV}$, and a Maxwell-Boltzmann energy distribution with a FWHM of $1 \mathrm{meV}$.

The objective lens setting remains constant as the imaging mode and FoV are modified. This lens magnifies the image of the sample by $\mathrm{M} \sim 8.5 \times$ onto the center of the field lens. The purpose of the field lens and the remaining components, the transfer and the diffraction lenses, is to properly map the sample and diffraction planes onto the principal planes of the prism.

In image mode, the diffraction lens remains de-excited. Here, the field lens maps the diffraction pattern onto the center of the transfer lens, which is also the prism slit plane; and the transfer lens maps the center of the field lens onto the achromatic plane. The overall magnification of the objective arm, from the sample to the achromatic plane, is $\mathrm{M} \sim 17 \times$.

In diffraction mode, the diffraction lens is excited along with the remaining lenses in order to swap the map of

\begin{tabular}{ccc}
\hline FoV & Blur & Distortion \\
\hline Small & $(85 \mathrm{~nm}, \sim 0.5$ pixel $)$ & $(80 \mathrm{~nm}, \sim 0.5$ pixel $)$ \\
Large & $(2.7 \mu \mathrm{m}, \sim 1.5$ pixels $)$ & $(3.5 \mu \mathrm{m}, \sim 2$ pixels $)$ \\
\hline
\end{tabular}

TABLE I: Optical performance of the objective lens evaluated in the field lens

\begin{tabular}{ccc}
\hline Mode,FoV & Blur & Distortion \\
\hline Image,Small & $(230 \mathrm{~nm}, \sim 0.7$ pixel $)$ & $(150 \mathrm{~nm}, \sim 0.4$ pixel $)$ \\
Image,Large & $(5.5 \mu \mathrm{m}, \sim 1.6$ pixels $)$ & $(8 \mu \mathrm{m}, \sim 2.4$ pixels $)$ \\
Diffraction,Small & $(26 \mathrm{~nm}, \sim 0.7$ pixel $)$ & $(40 \mathrm{~nm}, \sim 1$ pixel $)$ \\
Diffraction,Large & $(0.8 \mu \mathrm{m}, \sim 2$ pixels $)$ & $(1.2 \mu \mathrm{m}, \sim 3$ pixels $)$ \\
\hline
\end{tabular}

TABLE II: Optical performance of the entire objective arm evaluated in the prism principal planes

the sample and the diffraction pattern in the principal planes of the prism. Here, the field lens maps the diffraction plane onto the center of the diffraction lens, which is subsequently mapped by the transfer lens onto the prism achromatic plane; and the diffraction lens maps the image of the sample in the field lens onto the center of the transfer lens, which coincides with the prism slit plane. The overall magnification of the objective arm, from the sample to the last image plane prior to the prism, located at the center of the transfer lens is $\mathrm{M} \sim 2 \times$.

In a well-designed system, the aberrations of the objective lens dominate. Hence, the geometric-optical blur of the system as measured in equivalent pixels is expected to be independent of the imaging mode. This insensitivity is demonstrated by the values in tables I and II, which compare the optical blur and distortion values resulting from the objective lens alone with those from the entire objective arm in the two modes and for the two FoVs. The performance of the objective lens is evaluated in the field lens; the performance of the entire objective arm is evaluated in the principal planes of the prism: the achromatic plane in image mode; and the slit plane in diffraction mode. The equivalent pixel values are derived by properly magnifying the pixel values at the sample.

\section{B. Prism}

The prism simulations for the path from the objective arm to the detector were executed for both modes of operation and for both FoVs to ensure that this optical element behaved ideally as prescribed, i.e. as a stigmatic round lens. In addition to bending the beam by $90^{\circ}$, the prism introduces an energy dispersion in the slit plane, which is located $104.6 \mathrm{~mm}$ (approximately one Larmor diameter) from the prism center. For a $3 \mathrm{keV}$ beam, the dispersion is $\sim 17.6 \mu \mathrm{m} / \mathrm{V}$.

The optical blur induced by the aberrations as the beam passes through the prism was calculated for a starting angle of $500 \mathrm{mrad}$ at the sample and evaluated at the center of the prism - the virtual location of the achromatic plane. The value of the blur and distortion are 


\begin{tabular}{ccc}
\hline Mode,FoV & Blur & Distortion \\
\hline Image,Small & $(130 \mathrm{~nm}, \sim 0.4$ pixel $)$ & $(16 \mathrm{~nm}, \sim 0.05$ pixel $)$ \\
Image,Large & $(1.6 \mu \mathrm{m}, \sim 0.5$ pixel $)$ & $(2.5 \mu \mathrm{m}, \sim 0.7$ pixel $)$ \\
Diffraction,Small & $(1.6 \mu \mathrm{m}, \sim 0.4$ pixel $)$ & $(3.3 \mu \mathrm{m}, \sim 1$ pixel $)$ \\
Diffraction,Large & $(12.3 \mu \mathrm{m}, \sim 3$ pixels $)$ & $(3.5 \mu \mathrm{m}, \sim 1$ pixel $)$ \\
\hline
\end{tabular}

TABLE III: Optical performance of the prism

quoted at the corner of the field of view, where they are maximum, and compared to the pixel size derived from a detector with 500 pixels on each side.

In image mode, the linear and angular magnifications in the objective arm, from the sample to the achromatic plane, are equal to $\sim 17 \times$ and $\sim 0.00034 \times$, respectively. Hence, the size of the image inside the prism is at most $\sim 1.7 \mathrm{~mm}$, which is less than half of the gap distance of $5 \mathrm{~mm}$ between the two plates, rendering field aberrations negligible; and the convergence angle in the prism is approximately $0.17 \mathrm{mrad}$, well below the threshold of $\sim 1$ mrad, rendering axial aberrations negligible.

In diffraction mode, the angular pattern of the sample is magnified by $6 \times$ onto the prism achromatic plane. At the center of the prism, there is a field aperture (oriented at $45^{\circ}$ ) of $\sim 2 \mathrm{~mm}$ to limit the field aberrations. At this pattern size, the pixel size in the achromatic plane is 4 $\mu \mathrm{m}$. The convergence angle can be estimated by the ratio of the corner of the image in the slit plane to the distance between the slit and the prism center. Noting that the sample is magnified by $2 \times$ in the slit plane, the convergence angles for the two extreme FoVs are estimated as $\sim 0.13$ and $\sim 1.3$ mrad.

The optical blur and distortion resulting from the prism optics are shown in Table III. In both modes and for the range of FoVs, the spot size remains at or below 3 pixels, which is congruent with the Nyquist criterion.

\section{COULOMB INTERACTIONS}

Finally, the impact of electron Coulomb interactions on the optical performance is examined. Ionic contributions only influence the creation of the cold electrons, as discussed before. In particular, the increase in the beam energy spread with increasing beam current is calculated for the two modes and boundary FoVs for total emitted currents of $100 \mathrm{pA}, 1 \mathrm{nA}$, and $10 \mathrm{nA}$. For this calculation, the beam path from the source to the detector is separated into the illumination path, which extends from the source to the back-focal plane of the objective lens; and the projection path, which extends from the sample to the prism achromatic plane. The increase in the energy spread in the two paths is then summed in quadrature to obtain an estimate of the total energy spread.

The simulations show that the impact of the Coulomb interactions is mostly dependent on the total beam path and practically insensitive to the mode. In other words, the longer the electron path, the more chance that Coulomb interactions will dominate. The illumination

\begin{tabular}{cccc}
\hline Mode, FoV & $100 \mathrm{pA}$ & $1 \mathrm{nA}$ & $10 \mathrm{nA}$ \\
\hline Image, Small & 2.06 & 2.08 & 19.9 \\
Image, Large & 2.06 & 2.08 & 17.9 \\
Diffraction, Small & 2.06 & 2.08 & 19.9 \\
Diffraction, Large & 2.06 & 2.08 & 20.9 \\
\hline
\end{tabular}

TABLE IV: Final energy spread FWHM in meV in the illumination path for a range of beam currents.

path length is $640 \mathrm{~mm}$ long; the projection path length is mode dependent, equal to $235 \mathrm{~mm}$ in image mode and $340 \mathrm{~mm}$ diffraction mode.

The results for the illumination path in diffraction mode are illustrated in fig. 11. The figure shows the final electron energy distribution at the back-focal plane of the objectives lens for the three beam currents for the large and small field of view. Here, the small contribution in the beam path of about $20 \mathrm{~mm}$ from the objective back-focal plane to the sample has been neglected. The increase in the energy spread FWHM for the illumination and projection paths are summarized in Tables IV and V.

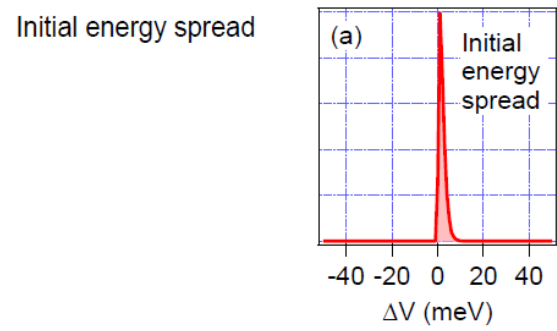

Final energy spread
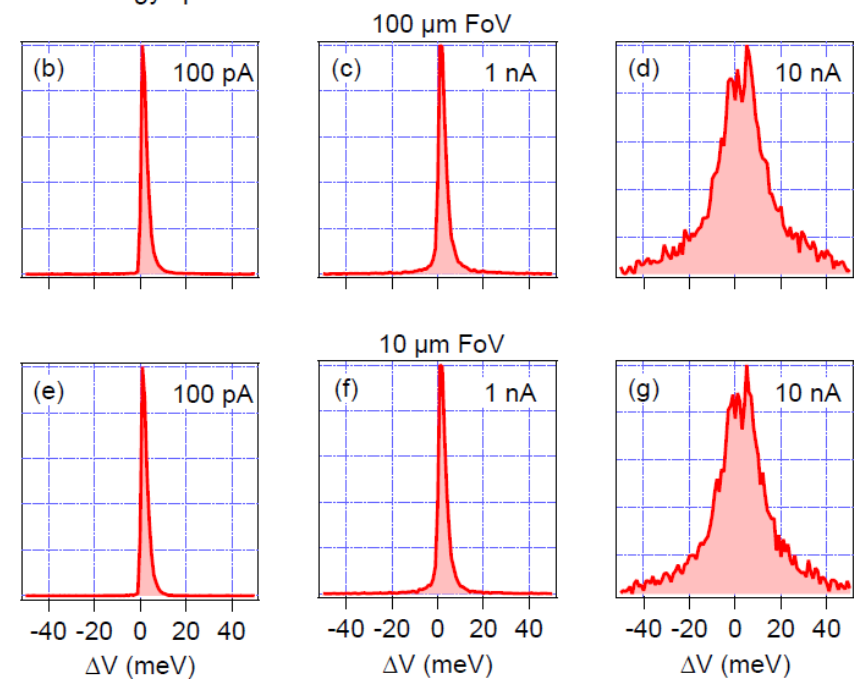

FIG. 11: (a) Initial energy spread of electron beam. Final electron energy spread distribution at the back focal plane of the objective lens for a range of beam currents in the diffraction mode for a large (b-d) and small (e-g) field of view.

It is evident that the increase in the FWHM of the energy spread due to Coulomb interactions is practically 


\begin{tabular}{cccc}
\hline Mode, FoV & $100 \mathrm{pA}$ & $1 \mathrm{nA}$ & $10 \mathrm{nA}$ \\
\hline Image, Small & 1.08 & 1.09 & 6.03 \\
Image, Large & 1.08 & 1.09 & 6.02 \\
Diffraction, Small & 1.08 & 1.09 & 8.01 \\
Diffraction, Large & 1.08 & 1.09 & 8.01 \\
\hline
\end{tabular}

TABLE V: Final energy spread FWHM in meV due to the objective optics for a range of beam currents.

negligible for beam currents up to $1 \mathrm{nA}$. However, at 10 $\mathrm{nA}$, the Coulomb induced energy spreads increase by $\sim$ $10 \times$ and $\sim 6-8 \times$ in the illumination and projection paths, respectively. These results are to all intents and purposes insensitive to the field of view and to the mode. The total spread, as derived via quadrature, is therefore essentially what is presented in Table IV, i.e. it is the length of the illumination path which determines the contribution of the Coulomb interaction.

\section{CONCLUSION}

The design of a HREELM column has been presented. The HREELM is similar to a LEEM column operating at a $3 \mathrm{kV}$ beam energy with an integrated imaging highresolution analyzer (ToF or HSA), designed for meV resolution. The full column will be comprised of magnetic lenses, a beam separator based on a magnetic prism ar- ray, and an electrostatic cold electron gun. A unique feature of the HREELM column is its capability to switch between modes that transfer either the image of the sample or its diffraction pattern through the prism achromatic plane. This allows for a dispersion-free image or diffraction pattern of the sample, while maintaining flexibility in operating the LEEM instrument.

The optics of the various components of the column were first analyzed separately to first order to determine their excitations and focusing properties. This analysis was followed by a calculation of the aberrations and of the impact of Coulomb interactions in the column. The calculations demonstrate that the column can image the sample or its momentum pattern with a resolution and distortion that meets the Nyquist criterion for fields of views in the range of $10-100 \mu \mathrm{m}$. The overall energy resolution is not limited by the electron optics but solely by the source parameters. The operating beam current in the electron optics column is found to be limited to 1 $\mathrm{nA}$ by the impact of Coulomb interactions.

\section{Acknowledgments}

The authors acknowledge the financial support from the Fond Unique Interministériel (IAPP-FUI-22: COLDFIB), the ANR-DFG 2014 HREELM and Scho341/14-1 project.
[1] M. Holt, Z. Wu, H. Hong, P. Zschack, P. Jemian, J. Tischler, H. Chen, and T. C. Chiang. Determination of phonon dispersions from x-ray transmission scattering: he example of silicon. Physical Review Letters, 83(16):3317-3319, 1999.

[2] H. Ibach and D. L. Mills. Electron energy loss spectroscopy and surface vibrations. Academic press, 1982.

[3] F. S. Tautz and J. A. Schaefer. Ultimate resolution electron energy loss spectroscopy at $\mathrm{H} / \mathrm{Si}(100)$ surfaces. Journal of Applied Physics, 84(12):6636-6643, 1998.

[4] T. Conard, L. Philippe, P. A. Thiry, P. Lambin, and R. Caudano. Electron energy-loss spectroscopy and dynamics of SrTiO3(100). Surface Science, 287-288(PART 1):382-385, 1993.

[5] P. Verma. Tip-Enhanced Raman Spectroscopy: Technique and Recent Advances. Chemical Reviews, 117(9):6447-6466, 2017.

[6] W Telieps and E Bauer. An analytical reflection and emission UHV surface electron microscope. Ultramicroscopy, 17(1):57-65, 1985.

[7] E. Bauer. Low energy electron microscopy of surfaces. Reports on Progress in Physics, 57:895-938, 1994.

[8] R. M. Tromp, J. B. Hannon, A. W. Ellis, W. Wan, A. Berghaus, and O. Schaff. A new aberration-corrected, energy-filtered LEEM/PEEM instrument. I. Principles and design. Ultramicroscopy, 110(7):852-61, 2010.

[9] O. L. Krivanek, J. P. Ursin, N. J. Bacon, G. J. Corbin, N. Dellby, P. Hrncirik, M. F. Murfitt, C. S. Own, and Z. S. Szilagyi. High-energy-resolution monochromator for aberration-corrected scanning transmission electron microscopy/electron energy-loss spectroscopy. Philosophical Transactions of the Royal Society A: Mathematical, Physical and Engineering Sciences, 367(1903):3683-3697, 2009.

[10] O. L. Krivanek, N. Dellby, J. A. Hachtel, J.-C. Idrobo, M. T. Hotz, B. Plotkin-Swing, N. J. Bacon, A. L. Bleloch, G. J. Corbin, M. V. Hoffman, et al. Progress in ultrahigh energy resolution EELS. Ultramicroscopy, 2018.

[11] R. Ahluwalia, N. Ng, A. Schilling, R. G. P. McQuaid, D. M. Evans, J. M. Gregg, D. J. Srolovitz, and J. F. Scott. Manipulating ferroelectric domains in nanostructures under electron beams. Phys. Rev. Lett., 111:165702, Oct 2013.

[12] M. Mukai, E. Okunishi, M. Ashino, K. Omoto, T. Fukuda, A. Ikeda, K. Somehara, T. Kaneyama, T. Saitoh, T. Hirayama, and Y. Ikuhara. Development of a monochromator for aberration-corrected scanning transmission electron microscopy. Microscopy (Oxford, England), 64(3):151-158, 2015.

[13] O. L. Krivanek, J. P. Ursin, N. J. Bacon, G. J. Corbin, N. Dellby, P. Hrncirik, M. F. Murfitt, C. S. Own, and Z. S. Szilagyi. High-energy-resolution monochromator for aberration-corrected scanning transmission electron microscopy/electron energy-loss spectroscopy. Philosophical transactions. Series A, Mathematical, physical, and engineering sciences, 367(1903):3683-3697, 2009.

[14] K. Kimoto. Practical aspects of monochromators developed for transmission electron microscopy. Microscopy 
(Oxford, England), 63(5):337-344, 2014.

[15] E. Essers, G. Benner, T. Mandler, S. Meyer, D. Mittmann, M. Schnell, and R. Höschen. Energy resolution of an omega-type monochromator and imaging properties of the mandoline filter. Ultramicroscopy, 110(8):971-980, 2010.

[16] F. S. Hage, R. J. Nicholls, J. R. Yates, D. G. McCulloch, T. C. Lovejoy, N. Dellby, O. L. Krivanek, K. Refson, and Q. M. Ramasse. Nanoscale momentum-resolved vibrational spectroscopy. Science advances, 4(6):eaar7495, 2018.

[17] A. C. Gallagher and G. York. A photoionization source of monoenergetic electrons. Review of Scientific Instruments, 45(5):662-668, 1974.

[18] R. F. Egerton. Electron energy-loss spectroscopy in the electron microscope. Springer Science \& Business Media, 2011.

[19] H. Ibach. Electron energy loss spectrometers: the technology of high performance, volume 63. Springer, 2013.

[20] C. Tusche, A. Krasyuk, and J. Kirschner. Spin resolved bandstructure imaging with a high resolution momentum microscope. Ultramicroscopy, 159:520-529, 2015.

[21] K. Medjanik, O. Fedchenko, S. Chernov, D. Kutnyakhov, M. Ellguth, A. Oelsner, B. Schönhense, T. R.F. Peixoto, P. Lutz, C. H. Min, F. Reinert, S. Däster, Y. Acremann, J. Viefhaus, W. Wurth, H. J. Elmers, and G. Schönhense. Direct 3D mapping of the Fermi surface and Fermi velocity. Nature Materials, 16(6):615-621, 2017.

[22] B. Schönhense, K. Medjanik, O. Fedchenko, S. Chernov, M. Ellguth, D. Vasilyev, A. Oelsner, J. Viefhaus, D. Kutnyakhov, W. Wurth, H. J. Elmers, and G. Schönhense. Multidimensional photoemission spectroscopy - The space-charge limit. New Journal of Physics, 20(3), 2018.

[23] O. Fedchenko, S. Chernov, A. McCulloch, M. VielleGrosjean, D. Comparat, and G. Schönhense. Extraction dynamics of electrons from magneto-optically trapped atoms. Applied Physics Letters, 111(2):1-6, 2017.

[24] D. A. Harmin. Analytical study of quasidiscrete stark levels in Rydberg atoms. Physical Review A, 30(5):24132428, 1984.

[25] R. E. Kennerly, R. J. van Brunt, and A. C. Gallagher. High-resolution measurement of the helium 1s2s2s2 resonance profile. Physical Review A, 23(5):2430-2442, 1981.

[26] J. Bömmels, E. Leber, A. Gopalan, J. M. Weber, S. Barsotti, M.-W. Ruf, and H. Hotop. Energy broadening due to photoion space charge in a high resolution laser photoelectron source. Review of Scientific Instruments, 72(11):4098-4105, 2001.
[27] J. Bömmels, K. Franz, T. H. Hoffmann, A. Gopalan, O. Zatsarinny, K. Bartschat, M.-W. Ruf, and H. Hotop. Low-lying resonances in electron-neon scattering: Measurements at 4- mev resolution and comparison with theory. Physical Review A, 71(1):012704, 2005.

[28] T. Ikegami. Angular distribution measurement of cesium atomic beam from long tube collimators. Japanese journal of applied physics, 33(8R):4795, 1994.

[29] B. Knuffman, A. V. Steele, and J. J. McClelland. Cold atomic beam ion source for focused ion beam applications. Journal of Applied Physics, 114(4):044303, 2013.

[30] V. Carrat, C. Cabrera-Gutiérrez, M. Jacquey, J. Tabosa, B. Viaris de Lesegno, and L. Pruvost. Long-distance channeling of cold atoms exiting a 2D magneto-optical trap by a Laguerre-Gaussian laser beam. Optics letters, 39(3):719-722, 2014.

[31] Y. Bruneau, G. Khalili, P. Pillet, and D. Comparat. Guided and focused slow atomic beam from a 2 dimensional magneto optical trap. The European Physical Journal D, 68(4):92, 2014.

[32] X. Yan, C. Wu, J. Huang, J. Zhang, and L. Wang. Velocity-tunable cold Cs atomic beam from a magnetooptical trap. Chinese Optics Letters, 15(4):040202, 2017.

[33] G. ten Haaf, T. C. H. de Raadt, G. P. Offermans, J. F. M. van Rens, P. H. A. Mutsaers, E. J. D. Vredenbregt, and S. H. W. Wouters. Direct magneto-optical compression of an effusive atomic beam for application in a highresolution focused ion beam. Physical Review Applied, 7(5):054013, 2017.

[34] L. Kime, A. Fioretti, Y. Bruneau, N. Porfido, F. Fuso, M. Viteau, G. Khalili, N. Šantić, A. Gloter, B. Rasser, P. Sudraud, P. Pillet, and D. Comparat. High-flux monochromatic ion and electron beams based on lasercooled atoms. Physical Review A, 88:033424, September 2013.

[35] A. J. McCulloch, R. W. Speirs, J. Grimmel, B. M. Sparkes, D. Comparat, and R. E. Scholten. Field ionization of Rydberg atoms for high-brightness electron and ion beams. Physical Review A, 95(6):063845, 2017.

[36] E. Moufarej, M. Vielle-Grosjean, G. Khalili, A. J. McCulloch, F. Robicheaux, Y. J. Picard, and D. Comparat. Forced field ionization of Rydberg states for the production of monochromatic beams. Physical Review A, 95(4), 2017.

[37] M. Mankos, V. Spasov, and E. Munro. Principles of Dual-Beam Low-Energy Electron Microscopy, volume 161. Elsevier Inc., 1 edition, 2010. 